\title{
Another topological proof of the Fundamental Theorem of Algebra
}

\author{
J.M. Almira, M. Jiménez, N. Del Toro* \\ J.M. Almira obtained his Ph.D. on approximation spaces at La Laguna University in \\ 1999. Presently, he is associate professor at the Department of Mathematics of Jaén \\ University. His main interests are: approximation theory, differential equations, new \\ proofs of classical results, and the history of mathematics. \\ M. Jiménez graduated at Granada University in 1989. He also obtained a degree \\ in management sciences at U.N.E.D. University in 2001. Presently, he works with \\ J.M. Almira on his $\mathrm{Ph} . \mathrm{D}$. thesis. He is interested in mathematical modeling and \\ optimization techniques. \\ N. Del Toro graduated at La Laguna University in 1999. Presently, she has a research \\ grant from Junta de Andalucía to complete her Ph.D. thesis on approximation theory.
}

\section{Introduction}

The Fundamental Theorem of Algebra claims that every polynomial $p(z) \in \mathbb{C}[z]$ can be decomposed as a product of linear factors, $p(z)=c \prod_{i=1}^{n}\left(z-\alpha_{i}\right)$. Now, if we assume that $p(z)$ is a monic polynomial, then $c=1$ and

$$
p(z)=z^{n}-a_{1}\left(\alpha_{1}, \ldots, \alpha_{n}\right) z^{n-1}+a_{2}\left(\alpha_{1}, \ldots, \alpha_{n}\right) z^{n-2}+\cdots+(-1)^{n} a_{n}\left(\alpha_{1}, \ldots, \alpha_{n}\right),
$$

Allen Lesern wird der Fundamentalsatz der Algebra bekannt sein. Er besagt, dass jedes Polynom $P=P(z)$ über dem Körper der komplexen Zahlen mindestens eine komplexe Nullstelle hat. Besitzt $P$ den Grad $n$, so ergibt sich daraus sofort, dass $P$ (mit Vielfachheiten gezählt) genau $n$ komplexe Nullstellen hat. Die Bestimmung der Nullstellen von Polynomen spielte in der Entwicklung der Algebra eine wichtige Rolle. Allerdings gelang es erst N.H. Abel zu beweisen, dass die Nullstellen eines Polynoms vom Grad $n>4$ in der Regel nicht durch Radikale darstellbar sind. Damit musste zum Beweis des Fundamentalsatzes nach neuen Ideen gesucht werden. Neben den Beweisen von C.F. Gauss wird der Fundamentalsatz heute sehr oft als elegante Anwendung aus dem Satz von Liouville in der Funktionentheorie gefolgert. Im vorliegenden Beitrag geben die Autoren einen ebenfalls eleganten Beweis des Fundamentalsatzes, der auf einfachen Ergebnissen der Topologie beruht.

*) Research partially supported by Junta de Andalucía, Grupo de Investigación "Aproximación y Métodos Numéricos" FQM-0178. 
where

$$
a_{k}\left(\alpha_{1}, \ldots, \alpha_{n}\right)=\sum_{1 \leq i_{1}<i_{2}<\cdots<i_{k} \leq n} \alpha_{i_{1}} \alpha_{i_{2}} \ldots \alpha_{i_{k}}
$$

are the elementary symmetric functions. Hence the next result holds:

Theorem 1 The following claims are equivalent:

(A) The Fundamental Theorem of Algebra

(B) The map $\sigma^{n}: \mathbb{C}^{n} \rightarrow \mathbb{C}^{n}$ given by

$$
\sigma^{n}\left(\alpha_{1}, \ldots, \alpha_{n}\right)=\left(a_{1}\left(\alpha_{1}, \ldots, \alpha_{n}\right), a_{2}\left(\alpha_{1}, \ldots, \alpha_{n}\right), \ldots, a_{n}\left(\alpha_{1}, \ldots, \alpha_{n}\right)\right)
$$

is onto for all $n \in \mathbb{N}$.

Proof. Let $\mathbf{a}=\left(a_{1}, \ldots, a_{n}\right) \in \mathbb{C}^{n}$ be arbitrarily chosen and set

$$
p_{\mathbf{a}}(z):=z^{n}+\sum_{k=1}^{n}(-1)^{k} a_{k} z^{n-k} .
$$

It follows from the Fundamental Theorem of Algebra that $p_{\mathbf{a}}(z)=\prod_{i=1}^{n}\left(z-\alpha_{i 0}\right)$ for a certain choice of complex numbers $\left\{\alpha_{i 0}\right\}_{i=1}^{n} \subset \mathbb{C}$. Hence $\mathbf{a}=\sigma^{n}\left(\alpha_{10}, \ldots, \alpha_{n 0}\right)$ and (A) implies (B). On the other hand, let $p(z)=z^{n}+\sum_{k=1}^{n}(-1)^{k} a_{k} z^{n-k}$ be arbitrarily chosen. Then there exists a certain point $\alpha=\left(\alpha_{10}, \ldots, \alpha_{n 0}\right) \in \mathbb{C}^{n}$ such that $\left(a_{1}, \ldots, a_{n}\right)=\sigma^{n}(\alpha)$. This implies the identity $p(z)=\prod_{i=1}^{n}\left(z-\alpha_{i 0}\right)$, which proves (A).

The main goal of this note is to give an elementary proof of (B) of the theorem above. For the proof we will need to use Brouwer's Theorem of Invariance of Domain (see [2, Theorem 36.5 , p. 207]) and a certain separation property of $\mathbb{R}^{n}$ :

Theorem 2 (Brouwer) Let us assume that $f: \Omega \subset \mathbb{R}^{n} \rightarrow \mathbb{R}^{n}$ is a continuous injective map, and $\boldsymbol{\Omega}$ is an open subset of $\mathbb{R}^{n}$. Then $f(\boldsymbol{\Omega})$ is an open subset of $\mathbb{R}^{n}$.

Theorem 3 Let $M \subset \mathbb{R}^{n}$ be an embedded submanifold of $\mathbb{R}^{n}$ of dimension $\leq n-2$, and let $\Delta$ be a subset of $M$. Then $\Delta \subset \mathbb{R}^{n}$ does not separate $\mathbb{R}^{n}$ (we say that the set $\Delta \subset \mathbb{R}^{n}$ separates $\mathbb{R}^{n}$ if $\mathbb{R}^{n} \backslash \Delta$ is not arcwise connected).

Proof. Recall that the $k$-manifold $M \subset \mathbb{R}^{n}$ is embedded in $\mathbb{R}^{n}$ if and only if for each $\mathbf{x} \in M$ there exists a bounded neighborhood $U_{\mathbf{x}}$ of $\mathbf{x}$ in $\mathbb{R}^{n}$ and a homeomorphism $\varphi_{\mathbf{x}}: \mathbf{C}_{n} \rightarrow U_{\mathbf{x}}$ such that $\varphi_{\mathbf{x}}\left(\mathbf{0}_{n}\right)=\mathbf{x}$ and $M \cap U_{\mathbf{x}}=\varphi_{\mathbf{x}}\left(\mathbf{C}_{n}^{k}\right)$, where $\mathbf{C}_{n}=[-1,1]^{n}, \mathbf{0}_{n}$ denotes the origin of coordinates of $\mathbb{R}^{n}$ and $\mathbf{C}_{n}^{k}=[-1,1]^{k} \times\left\{\mathbf{0}_{n-k}\right\}$. If $k \leq n-2$ then $\mathbf{C}_{n} \backslash \mathbf{C}_{n}^{k}$ is arcwise connected, so that

$$
\varphi_{\mathbf{x}}\left(\mathbf{C}_{n} \backslash \mathbf{C}_{n}^{k}\right)=U_{\mathbf{x}} \backslash\left(M \cap U_{\mathbf{x}}\right)
$$

is arcwise connected. It follows from the fact that $\mathbb{R}^{n}$ has a numerable dense subset that there exists a sequence $\left\{\mathbf{x}_{n}\right\}_{n=1}^{\infty} \subset M$ such that

$$
\left(U_{\mathbf{x}_{n}} \backslash\left(M \cap U_{\mathbf{x}_{n}}\right)\right) \cap\left(U_{\mathbf{x}_{n+1}} \backslash\left(M \cap U_{\mathbf{x}_{n+1}}\right)\right) \neq \emptyset
$$


for all $n$, and $M \subset \bigcup_{n=1}^{\infty} U_{\mathbf{x}_{n}}$. Hence

$$
T(M)=\bigcup_{n=1}^{\infty}\left(U_{\mathbf{x}_{n}} \backslash\left(M \cap U_{\mathbf{x}_{n}}\right)\right)
$$

is arcwise connected.

We may assume without loss of generality that $\Delta=M$, since if $M$ does not separate $\mathbb{R}^{n}$ then $\Delta$ cannot separate $\mathbb{R}^{n}$. Let $\mathbf{a}, \mathbf{b} \in \mathbb{R}^{n} \backslash M$ be arbitrarily chosen and let $\alpha:[0,1] \rightarrow \mathbb{R}^{n}$ be a path with end points $\{\mathbf{a}, \mathbf{b}\}$. If $\alpha([0,1]) \cap M \neq \emptyset$ then we will find another path $\beta:[0,1] \rightarrow \mathbb{R}^{n}$ with $\beta([0,1]) \cap M=\emptyset$. With this idea in mind, let us consider the intersection

$$
\alpha([0,1]) \cap T(M) .
$$

It follows from compactness of $\alpha([0,1])$ that there exists $t_{0}, t_{1} \in(0,1), t_{0}<t_{1}$ such that $\alpha(t) \notin T(M)$ for all $t \in[0,1] \backslash\left[t_{0}, t_{1}\right]$ and $\alpha\left(t_{i}\right) \in T(M)$ for $i=0,1$. On the other hand, there exists a path $\eta:\left[t_{0}, t_{1}\right] \rightarrow T(M)$ such that $\eta\left(t_{i}\right)=\alpha\left(t_{i}\right), i=0,1$ (since $T(M)$ is arcwise connected). It follows that

$$
\beta(t)= \begin{cases}\eta(t) & \text { if } t \in\left[t_{0}, t_{1}\right] \\ \alpha(t) & \text { otherwise }\end{cases}
$$

is a path $\beta:[0,1] \rightarrow \mathbb{R}^{n}$ with $\beta([0,1]) \cap M=\emptyset$ and end points $\{\mathbf{a}, \mathbf{b}\}$.

We believe that there are several advantages of our focus with respect to other proofs of the Fundamental Theorem of Algebra based in algebraic topology: Firstly, the starting point is very clear and no trick is used (you know what you must do from the very beginning) and, as a consequence, the proof is very intuitive. On the other hand, this proof needs the same background than others which are based on the knowledge of the homology of spheres. There are also analytical proofs of the Fundamental Theorem of Algebra (e.g., the one based on Rouché's theorem) which only require some topological background.

\section{Proof of the main result}

In order to prove that $\sigma^{n}$ is onto, we need firstly to state several technical results:

Lemma 4 Let us assume that $\sigma^{n}(A)$ is a bounded subset of $\mathbb{C}^{n}$. Then $A$ is a bounded subset of $\mathbb{C}^{n}$.

Proof. Let us assume that $\sup _{\alpha \in A}\left\|\sigma^{n}(\alpha)\right\|_{\infty}:=\sup _{\alpha \in A} \max _{k \leq n}\left|a_{k}(\alpha)\right| \leq C$, and let $\alpha \in A$ be arbitrarily chosen. Then

$$
\left|\alpha_{k}^{n}+\sum_{i=1}^{n}(-1)^{i} a_{i}(\alpha) \alpha_{k}^{n-i}\right|=0 \text { for all } k \leq n \text {. Hence }\left|\alpha_{k}\right|^{n} \leq \sum_{i=1}^{n}\left|a_{i}(\alpha)\right|\left|\alpha_{k}\right|^{n-i} \text {. }
$$

If $\left|\alpha_{k}\right| \leq 1$ we do nothing. Otherwise

$$
\left|\alpha_{k}\right| \leq \sum_{i=1}^{n}\left|a_{i}(\alpha)\right|\left|\alpha_{k}\right|^{1-i} \leq \sum_{i=1}^{n}\left|a_{i}(\alpha)\right| \leq n C . \quad \text { Hence } \quad \sup _{\alpha \in A} \max _{k \leq n}\left|\alpha_{k}\right| \leq \max \{n C, 1\} .
$$

This ends the proof. 
Corollary 5 The map $\sigma^{n}$ is closed (i.e, it sends closed sets to closed sets).

Proof. Let us assume that $\mathbf{y}$ belongs to the closure of $\sigma^{n}(M)$ and $M$ is a closed subset of $\mathbb{C}^{n}$. Then there exists a sequence of points $\left\{\mathbf{x}_{n}\right\} \subset M$ such that $\sigma^{n}\left(\mathbf{x}_{n}\right) \rightarrow \mathbf{y}$. Hence $\sigma^{n}\left(\left\{\mathbf{x}_{n}\right\}_{n=1}^{\infty}\right)$ is a bounded subset of $\mathbb{C}^{n}$ and it follows from Theorem 2 that $\left\{\mathbf{x}_{n}\right\} \subset \mathbb{C}^{n}$ is bounded. Hence there exists a convergent subsequence $\left\{\mathbf{x}_{n_{k}}\right\} \rightarrow \mathbf{x} \in M$ (since $M$ is closed) and

$$
\sigma^{n}(\mathbf{x})=\lim _{k \rightarrow \infty} \sigma^{n}\left(\mathbf{x}_{n_{k}}\right)=\lim _{n \rightarrow \infty} \sigma^{n}\left(\mathbf{x}_{n}\right)=\mathbf{y} .
$$

This proves that $\mathbf{y} \in \sigma^{n}(M)$.

Lemma 6 Let us assume that $\alpha_{i} \neq \alpha_{j}$ for all $i \neq j$. Then there exists an open set $U=\stackrel{\circ}{U} \subset \mathbb{C}^{n}$ such that $\sigma_{\mid U}^{n}$ is one-to-one and $\left(\alpha_{1}, \ldots, \alpha_{n}\right) \in U$.

Proof. It is easy to prove that under these hypotheses, there exists a neighborhood of $\alpha=\left(\alpha_{1}, \ldots, \alpha_{n}\right)$ such that

$$
\left(\beta_{1}, \ldots, \beta_{n}\right) \in U \Rightarrow\left(\beta_{\theta(1)}, \ldots, \beta_{\theta(n)}\right) \notin U \text { for all } \theta \in \Sigma_{n} \backslash\{\mathrm{id}\} .
$$

Now, assume that $\sigma^{n}\left(\beta_{1}, \ldots, \beta_{n}\right)=\sigma^{n}\left(\beta_{1}^{*}, \ldots, \beta_{n}^{*}\right)$ and $\left(\beta_{1}, \ldots, \beta_{n}\right) \neq\left(\beta_{1}^{*}, \ldots, \beta_{n}^{*}\right)$ belong both to $U$. Then $p(z):=\prod_{i=1}^{n}\left(z-\beta_{i}\right)=\prod_{i=1}^{n}\left(z-\beta_{i}^{*}\right)$ is a polynomial of degree $n$ which vanishes on the set $\left\{\beta_{i}\right\}_{i=1}^{n} \cup\left\{\beta_{i}^{*}\right\}_{i=1}^{n}$. This implies that $\left(\beta_{1}^{*}, \ldots, \beta_{n}^{*}\right)=$ $\left(\beta_{\theta(1)}, \ldots, \beta_{\theta(n)}\right)$ for a certain $\theta \in \Sigma_{n} \backslash\{\mathrm{id}\}$, because of the divisibility properties of polynomials (which are proved as a consequence of the division algorithm of Euclid), a contradiction.

Corollary 7 Let $u$ s assume that $\alpha_{i} \neq \alpha_{j}$ for all $i \neq j$ and $i, j \in\{1, \ldots, n\}$. Then there exists an open set $U=\stackrel{\circ}{U} \subset \mathbb{C}^{n}$ such that $\left(\alpha_{1}, \ldots, \alpha_{n}\right) \in U, \sigma^{n}(U)$ is an open subset of $\mathbb{C}^{n}$, and $\sigma_{\mid U}^{n}: U \rightarrow \sigma^{n}(U)$ is a homeomorphism.

Proof. If we take $U$ as in the lemma above, then $\sigma_{\mid U}^{n}: U \rightarrow \sigma^{n}(U)$ is continuous, closed and bijective. This obviously implies that it is also open, hence it is a homeomorphism. Furthermore, the Theorem of Invariance of Domain claims that $\sigma^{n}(U)$ is an open subset of $\mathbb{C}^{n}$.

Lemma 8 Let $\mathbf{H}_{i, j}=\left\{\left(z_{1}, z_{2}, \ldots, z_{n}\right) \in \mathbb{C}^{n}: z_{i}-z_{j}=0\right\}$. Then

$$
\boldsymbol{\Gamma}:=\sigma^{n}\left(\mathbf{H}_{i, j}\right)=\sigma^{n}\left(\bigcup_{t<s} \mathbf{H}_{t, s}\right)
$$

for all $1 \leq i<j \leq n$.

Proof. Let $1 \leq t<s \leq n$ and $\alpha \in \mathbf{H}_{t, s}$ be arbitrarily chosen. Let $\theta \in \Sigma_{n}$ be such that $\theta(t)=i$ and $\theta(s)=j$. Then

$$
\sigma^{n}(\alpha)=\sigma^{n}\left(\alpha_{1}, \ldots, \alpha_{n}\right)=\sigma^{n}\left(\alpha_{\theta(1)}, \ldots, \alpha_{\theta(n)}\right) \in \sigma^{n}\left(\mathbf{H}_{i, j}\right) .
$$

This means that $\sigma^{n}\left(\mathbf{H}_{t, s}\right) \subset \sigma^{n}\left(\mathbf{H}_{i, j}\right)$ for all $t<s$. Hence $\sigma^{n}\left(\bigcup_{t<s} \mathbf{H}_{t, s}\right) \subset \sigma^{n}\left(\mathbf{H}_{i, j}\right)$, and the proof follows. 
Lemma $9 \mathbb{C}^{n} \backslash \bigcup_{t<s} \mathbf{H}_{t, s}$ and $\mathbb{C}^{n} \backslash \boldsymbol{\Gamma}$ are both open connected sets. Furthermore,

$$
\sigma^{n}\left(\mathbb{C}^{n} \backslash \bigcup_{t<s} \mathbf{H}_{t, s}\right) \subseteq \mathbb{C}^{n} \backslash \boldsymbol{\Gamma} .
$$

Proof. It is clear that both sets are open, since $\bigcup_{t<s} \mathbf{H}_{t, s}$ is closed, $\boldsymbol{\Gamma}=\sigma^{n}\left(\bigcup_{t<s} \mathbf{H}_{t, s}\right)$ and $\sigma^{n}$ is a closed map. If we write the equations which define $\mathbf{H}_{t, s}$ as a linear subspace of $\mathbb{R}^{2 n} \equiv \mathbb{C}^{n}$ (where we identify $z_{i}=x_{i}+\mathbf{i} y_{i}$ with the pair $\left(x_{i}, y_{i}\right)$ ), we have that $\mathbf{H}_{t, s}=\operatorname{ker} \mathbf{L}_{(t, s)}$, where

$$
\mathbf{L}_{(t, s)}\left(x_{1}, y_{1}, x_{2}, y_{2}, \ldots, x_{n}, y_{n}\right)=\left(x_{t}-x_{s}, y_{t}-y_{s}\right)
$$

Hence $\operatorname{dim} \mathbf{H}_{t, s}=2 n-2$ for all $t<s$, and this implies that $\bigcup_{t<s} \mathbf{H}_{t, s}$ does not separate $\mathbb{R}^{2 n}$. Hence $\mathbb{C}^{n} \backslash \bigcup_{t<s} \mathbf{H}_{t, s}$ is a connected set.

On the other hand, it follows from the identities $\boldsymbol{\Gamma}=\sigma^{n}\left(\mathbf{H}_{1,2}\right)$ and

$$
\begin{aligned}
(z+ & \alpha)^{2}\left(z^{n-2}+b_{1} z^{n-3}+\cdots+b_{n-2}\right) \\
= & z^{n}+\left(b_{1}+2 \alpha\right) z^{n-1}+\left(b_{2}+2 \alpha b_{1}+\alpha^{2}\right) z^{n-2} \\
& +\sum_{k=3}^{n-2}\left(b_{k}+2 \alpha b_{k-1}+\alpha^{2} b_{k-2}\right) z^{n-k}+2 \alpha b_{n-2} z+\alpha^{2} b_{n-2},
\end{aligned}
$$

that $\boldsymbol{\Gamma}$ is a subset of $\mathbf{M}=\left\{\mathbf{A}(\alpha) \cdot \mathbf{b}: \alpha \in \mathbb{C}, \mathbf{b}=\left(b_{1}, \ldots, b_{n-2}\right) \in \mathbb{C}^{n-2}\right\}$, where

$$
\mathbf{A}(\alpha)=\left(\begin{array}{ccclcc}
-1 & 0 & 0 & \cdots & 0 & 0 \\
2 \alpha & 1 & 0 & & \vdots & \vdots \\
-\alpha^{2} & -2 \alpha & -1 & \cdots & & \\
0 & \alpha^{2} & 2 \alpha & \cdots & & \\
0 & 0 & -\alpha^{2} & \cdots & (-1)^{n-3} & 0 \\
0 & 0 & 0 & & 2(-1)^{n-2} \alpha & (-1)^{n-2} \\
\vdots & \vdots & \vdots & & (-1)^{n-1} \alpha^{2} & 2(-1)^{n-1} \alpha \\
0 & 0 & 0 & \ldots & 0 & (-1)^{n} \alpha^{2}
\end{array}\right) \in \mathbf{M}_{n \times(n-2)}(\mathbb{C})
$$

for all $\alpha \in \mathbb{C}$. Now, $\operatorname{rank}(\mathbf{A}(\alpha))=n-2$ (as a complex matrix) for all $\alpha \in \mathbb{C}$. Hence $\mathbf{M}$ is a ruled submanifold of $\mathbb{C}^{n} \equiv \mathbb{R}^{2 n}$ of complex dimension equal to $n-1$, so that it has real dimension $2 n-2$. Hence $\mathbb{R}^{2 n} \backslash \Delta$ is arcwise connected for all $\Delta \subseteq \mathbf{M}$. This means that $\mathbb{C}^{n} \backslash \Gamma$ is arcwise connected.

Now, we prove the inclusion formula (1). Let us assume that $\sigma^{n}(\alpha) \in \boldsymbol{\Gamma}$. Then there exists a certain $\beta \in \mathbf{H}_{1,2}$ such that $\sigma^{n}(\alpha)=\sigma^{n}(\beta)$. But this implies that $\alpha=\left(\beta_{\theta(1)}, \ldots, \beta_{\theta(n)}\right)$ for a certain $\theta \in \Sigma_{n}$ (as in the proof of Lemma 4). Hence $\alpha \in \bigcup_{t<s} \mathbf{H}_{t, s}$. This ends the proof.

Now we are able to prove the main result of this paper: 
Theorem 10 The map $\sigma^{n}: \mathbb{C}^{n} \rightarrow \mathbb{C}^{n}$ is onto for all $n \in \mathbb{N}$.

Proof. It is clear that

$$
\sigma^{n}\left(\mathbb{C}^{n}\right)=\sigma^{n}\left(\mathbb{C}^{n} \backslash \bigcup_{t<s} \mathbf{H}_{t, s}\right) \cup \boldsymbol{\Gamma} .
$$

Hence we only need to prove that $\sigma^{n}\left(\mathbb{C}^{n} \backslash \bigcup_{t<s} \mathbf{H}_{t, s}\right)=\mathbb{C}^{n} \backslash \boldsymbol{\Gamma}$. Now, it follows from Corollary 5 and 7 that $\sigma^{n}\left(\mathbb{C}^{n} \backslash \bigcup_{t<s} \mathbf{H}_{t, s}\right)$ is a connected open subset of $\mathbb{C}^{n} \backslash \boldsymbol{\Gamma}$. On the other hand, if $\mathbf{y} \in \mathbb{C}^{n} \backslash \boldsymbol{\Gamma}$ is a point of the closure of $\sigma^{n}\left(\mathbb{C}^{n} \backslash \bigcup_{t<s} \mathbf{H}_{t, s}\right)$ in $\mathbb{C}^{n} \backslash \boldsymbol{\Gamma}$, then (as it was proved in Corollary 5), there exists a convergent sequence $\left\{\mathbf{x}_{n_{k}}\right\} \subset \mathbb{C}^{n} \backslash \bigcup_{t<s} \mathbf{H}_{t, s}$, such that $\left\{\mathbf{x}_{n_{k}}\right\} \rightarrow \mathbf{x} \in \overline{\mathbb{C}^{n} \backslash \bigcup_{t<s} \mathbf{H}_{t, s}}$,

$$
\sigma^{n}(\mathbf{x})=\lim _{k \rightarrow \infty} \sigma^{n}\left(\mathbf{x}_{n_{k}}\right)=\mathbf{y}
$$

But $\mathbf{y} \notin \boldsymbol{\Gamma}$ implies that $\mathbf{x} \in \mathbb{C}^{n} \backslash \bigcup_{t<s} \mathbf{H}_{t, s}$. Hence $\sigma^{n}\left(\mathbb{C}^{n} \backslash \bigcup_{t<s} \mathbf{H}_{t, s}\right)$ is a closed subset of $\mathbb{C}^{n} \backslash \boldsymbol{\Gamma}$. Now we use that $\mathbb{C}^{n} \backslash \boldsymbol{\Gamma}$ is connected to obtain that $\sigma^{n}\left(\mathbb{C}^{n} \backslash \bigcup_{t<s} \mathbf{H}_{t, s}\right)=\mathbb{C}^{n} \backslash \boldsymbol{\Gamma}$, which is what we wish to prove.

Remark 11 The proof uses that we are dealing with complex polynomials since otherwise the sets $\mathbf{H}_{i, j}$ would be hyperspaces in $\mathbb{R}^{n}$, so that $\mathbb{R}^{n} \backslash \bigcup_{t<s} \mathbf{H}_{t, s}$ could not be a connected set.

Remark 12 With the use of a little of complex analysis there are several more elementary proofs of the Fundamental Theorem of Algebra (see [1], [3]). The standard focus is to use Liouville's theorem. Another point of view (that usually does not appear in textbooks), very near to our proof, is as follows: first prove the open mapping theorem (i.e., that non-constant holomorphic functions are open maps), then prove by similar arguments to those given in Lemma 4 and Corollary 5, that $p(z)=z^{n}+\sum_{k=1}^{n} a_{k} z^{n-k}$ is a closed map for all choices of coefficients $a_{1}, \ldots, a_{n} \in \mathbb{C}$. Finally, you use that $\mathbb{C}$ is connected.

\section{References}

[1] Benjamin, F., Rosenberger, G.: The Fundamental Theorem of Algebra, Undergraduate texts in Math. Springer, 1997.

[2] Munkres, J.R.: Elements of Algebraic Topology, Addison-Wesley, 1984.

[3] Rudin, W.: Real and Complex Analysis, McGraw-Hill, 1974.

J.M. Almira, M. Jiménez, N. Del Toro

Departamento de Matemáticas

Universidad de Jaén

E.U.P. Linares

23700 Linares (Jaén), Spain

e-mail: jmalmira@ujaen.es 